\title{
Sediment risk analysis of urban drainage pipeline
}

\author{
WenPing $\mathrm{Li}^{1}$, WeiYi $\mathrm{He}^{1}$, Jin $\mathrm{Xu}^{2, *}$ \\ ${ }^{1}$ School of Management, Tianjin University of Technology, Tianjin, China \\ ${ }^{2}$ Department of Environmental Science and Safety Engineering, Tianjin University of Technology, Tianjin, China
}

\begin{abstract}
The urban water body is an important part of the urban infrastructure, and the urban drainage pipe is the core part of the urban water system, however, heavy metals and nutrients such as nitrogen and phosphorus in the sediments of urban drainage pipes have some risks, so it is important to analyze the risk of sediments in urban drainage pipes. This paper analyzes the risk and harm of sediment in drainage pipeline from three aspects: human health, water ecological safety and water environmental quality, it will lay a foundation for further study on the risk of sediment in drainage pipeline to water environment.
\end{abstract}

\section{Introduction}

With the rapid development of society, economy and cities, a large number of urban pollutants enter urban water bodies, causing most of the city's ditches, lakes and rivers to be polluted, urban water environment crisis has become one of the main factors restricting urban development[13]. The problems of urban water environment are becoming more and more serious, such as the serious shortage of water resources caused by over-exploitation of water resources, the serious pollution of rivers caused by over-discharge of industrial wastewater. Therefore, in order to effectively control urban water pollution, it is necessary to identify the source of water pollution.

Urban drainage system is a series of systems and facilities which collect and transport urban domestic sewage, industrial wastewater and surface runoff of rainfall to reduce the harm to urban water resources through timely removal, it is an important link in the process of urban water pollution control. As the core part of urban drainage system, urban drainage pipe is not only the urban drainage channel, but also an important way for non-point source pollution to enter the urban water body. At present, there are some problems such as sediment accumulation and pipe corrosion in drainage pipes of many cities in China. The investigation found that $60 \%$ of the drainage pipes in Beijing urban area had sediment, and $15 \%$ of the drainage pipes had large sediment amount, while nearly $75 \%$ of the drainage pipes in the old urban area on both sides of the Xinhepuyong River basin in Guangzhou had sediment in different degrees, and the deposition situation in the regions with more serious environmental pollution is more serious.

The sediment in the drainage pipe contains a lot of organic and toxic metals which are easy to be degraded. The deposition of the sediment will not only corrode the pipe, but also release a lot of pollutants accumulated in the sediment during overdischarge in rainy days into the natural water body, it is a serious threat to the water environment safety of the receiving water body and the health of the urban residents. Therefore, in order to prevent and control the further deterioration of urban water quality and ensure the healthy and sustainable development of urban water environment, it is urgent to strengthen the study on the sediment risk of urban drainage pipeline.

\section{Problems with the drainage system}

The drainage pipeline plays an important role in protecting the environment, reducing pollution, improving people's health and maintaining the normal life order of the city. However, due to planning, design, construction and other reasons, there are always a series of problems in the operation of urban drainage pipeline system.

\section{1 mixing of rain and pollution}

Theoretically speaking, the separate drainage system is more advanced than the combined drainage system, which causes less pollution to the receiving water environment. In China, urban development is very rapid, although the drainage system in the planning, design, construction and management of rapid progress, but the rain, sewage mixing phenomenon is still very serious. According to the investigation, the situation of drainage facilities in many areas of Wuhan is quite different from the planning. The phenomenon of mixing of rain and sewage is quite common. Almost all the storm water pipes investigated have sewage connections, and the vast majority of the storm water pipes also have rainwater connections, therefore, the rainwater and sewage pipes implemented according to the split-flow system have actually become double-row confluence pipes.

\footnotetext{
${ }^{*}$ Corresponding author: xujinhb@email.tjut.edu.cn
} 


\section{2 pipeline leakage}

Pipeline leakage is another important problem in drainage pipeline, the main reasons are as follows: (1) the bad foundation condition of pipeline will lead to uneven settlement of pipeline and foundation, water leakage at the joint, and pipeline fracture or joint crack. (2) the quality of the pipe is poor, there are cracks or local loose, poor impermeability, easy to produce leakage. (3) the joint packing and the construction quality are poor, the pipeline has the breakage or the joint crack under the external force action. (4) the construction quality of the inspection well is poor, and the well wall and the joint with its connecting pipe are leaking. (5) in addition, pipeline corrosion damage is also one of the reasons for pipeline leakage.

\section{3 clogged pipes}

Pipe clogging is usually caused by sediment deposition in the urban drainage system. There are many sources of solid particles on the urban surface, such as the decline of leaves, branches, soil, vehicles and the solid waste produced by people. Under the action of gravity and frictional resistance of pipe wall, the particles with large diameter and density will easily settle down to the bottom of pipe. It is also possible for small particles to gradually coalesce and grow as they collide with each other, thus settling the bottom of the pipe[4].

The drainage pipe system is an important infrastructure of the city, which plays an important role in maintaining the normal operation of the city. When the sediment in the drainage pipe reaches a certain degree, the transportation capacity of the pipe drops sharply, the rate at which sediment is deposited gradually increases, eventually causing a blockage in the pipe. The blockage of the drainage pipe will cause the sewage to overflow and pollute the environment. In addition, the sediment in the drainage pipeline is easy to cause corrosion and reduce the service life of the drainage pipeline system under the biochemical action.

\section{Source of sediment}

Urban drainage pipe is an important part of urban drainage system, which has the important function of collecting and conveying domestic sewage, industrial waste water and rainwater. There are two main sources of sediment in urban drainage pipes: one is the surface particulate matter accumulated on various water catchment surfaces in dry season, which flows into the drainage pipe network through the storm water outlet; The second is the deposition of suspended particulate matter in the sewer network itself.

The main sources of the surface particulate matter washed into the drainage pipes are: The roof, drainage, parking lot, highway and other sink water surface washed by rainwater and atmospheric deposition during the rain. The solid particulate matter in sewage pipe network comes from three aspects[5]: firstly, the small particle size residue of human excreta and organic particulate matter, which is the main source of sediment in sewage pipe; second is the kitchen, domestic waste in the large particle size residue and organic solid material; in addition, there are some paper, discarded clothing and other objects, such as objects, although not many, but the harm is very big, it is easy to cause pipeline blockage. Ahyerre et al [6] based on the physico-chemical properties of the channel sediments, they were divided into bottom coarse-grained sediments (Gross Bed Sediment, GBS) according to their distance from the bottom of the tube, the Organic Layer (OL) and the biomembrane (Biofilm) are three layers, as shown in Figure 1.

The pollutants in the sediments of urban drainage system are released into the receiving water body, which poses a serious threat to the safety of urban water environment. In addition, the study of risk assessment of water environmental safety from pollutant release will involve complicated processes such as its harmful effects on human health, water ecological safety and water environmental quality, therefore, it is necessary to study the sediment risk of urban drainage pipeline from all levels and aspects.

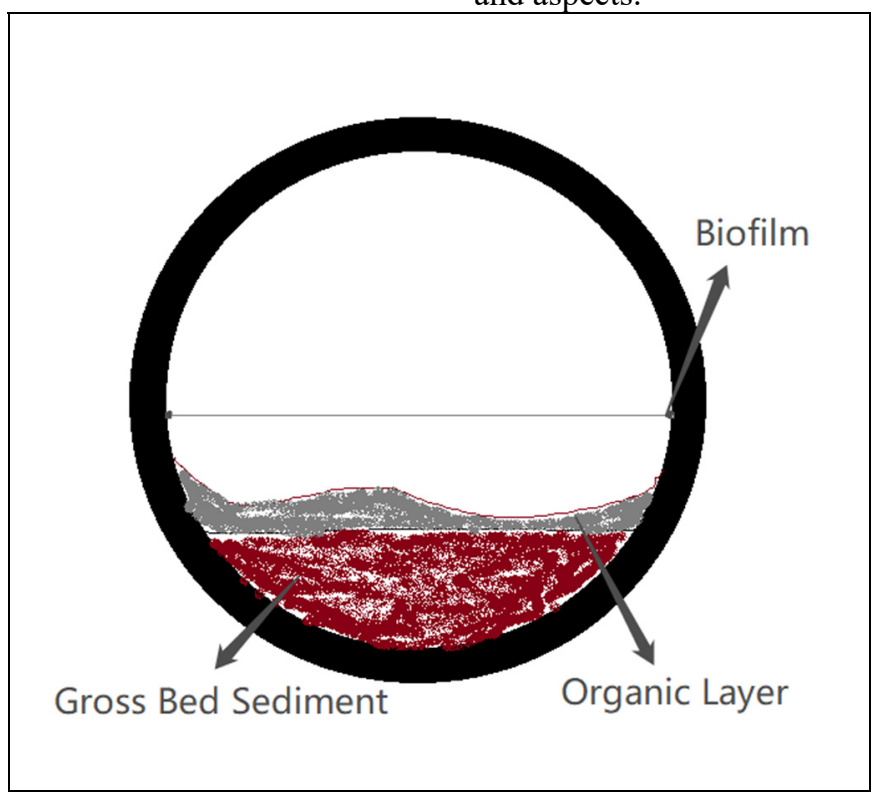

Fig. 1. Distribution of three types of sediments in the pipeline 


\section{Sediment is at risk}

\subsection{Human health}

There are many kinds of pollutants in the sediments in the drainage pipes, such as easily degradable organic compounds, nitrogen and phosphorus pollutants which easily cause algal bloom, refractory oils, lipids, toxic petroleum hydrocarbons (PHC) and polycyclic aromatic hydrocarbon and harmful heavy metals. Heavy metals are potentially harmful pollutants, which are concentrated in living organisms and become persistent pollutants, causing serious environmental problems. Heavy metal pollutants that enter the environment by various ways are not easy to dissolve, and migrate and circulate in the ecosystem by various ways, and may eventually enter the human body, causing serious harm. Heavy metals do great harm to human body, such as $\mathrm{Pb}, \mathrm{Hg}, \mathrm{Cd}$ and so on, they will cause great influence to human brain, nerve, vision and so on, and then do harm to human body health.

\subsection{Water ecological security}

The sediment pollution in the drainage pipe will be washed into the water body by the runoff during the rainstorm, which will form the impact pollution load, and then lead to the water ecological security problem and even more serious impact. As pollutants in the sediment of drainage pipes, nutrients such as nitrogen and phosphorus enter lakes, reservoirs and rivers in large quantities, and excessive input of nitrogen and phosphorus can lead to algal bloom. Yu Hang et al [7] expounded the harm caused by algal bloom from the aspects of ecological landscape, species diversity and stability of ecological system, human health and economic loss. The explosion of algae or aquatic plants in the water seriously hindered the photosynthesis of deep-seated plants, which led to the decrease of oxygen content in the water and the decrease of water transparency, in turn, fish and other organisms in the water due to lack of oxygen caused a large number of deaths. When the algal residue decomposes, more dissolved oxygen will be consumed, and in the process of decomposition, toxic and harmful substances will be produced, and other aquatic organisms will die in large numbers. The water body will be controlled by a single species of algae, and the biodiversity will be reduced, water pollution will be further aggravated and water ecological security will be seriously damaged.

\subsection{Water quality}

Urban rainstorm with high intensity and short duration will cause the overflow of drainage pipe. The overflow sewage contains various pathogenic microorganisms, organic and inorganic toxic and harmful substances, which pose a serious threat to urban water environment, destroy the water environment quality of the receiving water body. Studies have shown that about $80 \%$ of the total pollutant load from combined overflow is from the sediments in the drainage pipes[8]. The heavy metals in the pipeline sediment are discharged to the water body through many channels, and the heavy metals mainly exist, migrate and transform in the water as particles. The discharge of heavy metals far exceeds the water body's purification capacity, as a result, the composition and properties of water change, which has a great impact on the quality of water environment. In 2002, the concentration of total phosphorus (TP) in Huai River was 0.04-0.18 mg/l, and the concentration of total nitrogen (TN) was 0.94-5.4 $\mathrm{mg} / \mathrm{L}$, which was much higher than the standard of 0.02 $\mathrm{mg} / \mathrm{L}$ issued in China, the total nitrogen and phosphorus content of the Yellow River and the Yangtze River is still higher than the national standard, and the algal bloom is serious. After a large amount of plant nutrient elements such as nitrogen and phosphorus enter the water body, it will cause the phenomenon of excessive nutrient substance, algae multiplication, water quality deterioration, aquatic organism death, at the same time, it will also produce some toxic substances, which will seriously endanger the water environment quality.

\section{Conclusion}

The drainage system is figuratively compared to the lifeblood of a city. The urban drainage pipe system is responsible for the collection and discharge of rainwater, sewage and wastewater. It is the main way for pollutants to enter the urban water body, and it ensures the normal operation of the drainage system, it plays a very important role in protecting the environment, reducing pollution, improving people's health level and maintaining the normal order of city life. However, there are risks associated with urban sewer deposits, which contain heavy metals that can have a significant impact on the brain, vision, nerves, and so on; nutrients such as nitrogen and phosphorus can cause algal bloom, the water body is controlled by a single species of algae, which reduces the biodiversity, further aggravates the water environment pollution and seriously damages the water ecological security. Therefore, there are great risks to human health, water ecological safety and water environmental quality.

\section{Acknowledgement}

This work was sponsored in part by Tianjin Science and technology major special projects and engineering projects (18ZXSZSF00090), Ministry of Education Humanities and Social Sciences Research Planning Fund (18YJA630121), Tianjin Key R\&D Plan Achievements Transformation Relay Support Project (18YFJLCG00140), Tianjin Municipal Education Commission Social Science Major Project (2018JWZD33), Tianjin Science and Technology Major Special Project and Engineering Project (17ZXSTSF00020), Research and development and system integration application of "Internet +" sudden water environmental safety accident monitoring and early warning technology (19JCTPJC57100), Tianjin "Project + Team" key training special project (BIM-based digital twin building intelligent simulation and operation and maintenance management) 


\section{References}

1. L. Del Bello, Nature, 560, 149-150 (2018)

2. H.W. Huang, S.Y. Zeng, X. Dong, D. Li, Y. Zhang, M. He, P.F. Du, J. Environ. Manage, 231, 494-503 (2018)

3. D. Wu, Y.L. Su, H. Xi, X.Y. Chen, B. Xie, Water. Res, 158, 11-21 (2019)

4. W.E. Cotham, T.F. Bidleman, Environ. Sci. Technol, 29, 2782-2789 (1995)

5. R.M. Ashley, R.W Crabtree, Water. Sci. Technol, 25, 1-12 (2011)

6. M. Ahyerre, G. Chebbo, M. Saad, Urban Water, 2, 305-315. (2000)

7. R.M. Ashley, J.L. Bertrand-Krajewski, T. HvitvedJacobsen, M. Verbanck, IWA Publishing (2004)

8. R. Banasiak, R. Verhoeven, R. De Sutter, S. Tait, Water. Res, 39, 5221-5231 (2005) 